\title{
Improving the Reliability, Ease, and Efficiency of Section Staining in a Diagnostic Laboratory with the mPrep/g System for TEM Grid Processing
}

\author{
Craig Radi ${ }^{1}$ \\ ${ }^{1}$ Wisconsin Veterinary Diagnostic Laboratory, Madison, WI.
}

In a clinical diagnostic lab it is important to obtain results efficiently. With Transmission Electron Microscopy (TEM), a common problem is inconsistent staining of tissue sections that can lead to inefficiency. Poor staining can substantially delay diagnoses due to the time to prepare more sections, stain more grids, and then repeat TEM imaging. Most labs have a favorite method, with many using a variation of droplet staining [1]. We recently learned of an entirely new method based on mPrep/g (microscopy preparation for grids) capsules that can simultaneous stain 16 or more grids. In the present study we compare the mPrep/g method to our droplet method.

This study's droplet method used a pipet tip box covered with Parafilm that was slightly dimpled to provide a depression to hold stain droplets. Grids were placed section-side down on droplets of $2 \%$ aqueous Uranyl Acetate (UA), for 10 minutes, picked up with forceps, and rinsed with a water stream from a transfer pipette. They were then placed on droplets of Reynolds Lead Citrate (LC) for 10 minutes and covered. The grids were then rinsed with .002 Normal $\mathrm{NaOH}$, followed by a water stream rinse, blotted with filter paper, and stored until TEM examination. Two problems that come up using this method at times is damage to the sections as you pick up grid between staining and rinsing and having to add more rinses to the sections.

With the mPrep/g method, 1 or 2 grids are placed directly into $\mathrm{mPrep} / \mathrm{g}$ capsules at the microtome, into tapered grid slots that securely hold them even if a capsule is dropped. For staining, the capsules attach like pipette tips to lab pipettors. This study used a $200 \mu$ l 8 -channel Gilson Pipetman to enable staining of 16 grids in $8 \mathrm{mPrep} / \mathrm{g}$ capsules. Grids were stained from reagents held in a 96-well plate (Fig 1). The first row of the 96-well plate held $40 \mu$ of UA, the next 3 rows held $400 \mu \mathrm{l}$ of water, then $40 \mu \mathrm{l}$ of LC, then 3 more water rows with $400 \mu$ l of water. $8 \mathrm{mPrep} / \mathrm{g}$ capsules holding 2 grids each were attached to the 8-channel Pipetman, and dipped into the bottom of the UA reagent row. $35 \mu$ of UA was then drawn into the capsules and held for 10 minutes. The UA was then expelled back into this row, and the capsules/pipette were moved to the first water row. In this row, $40 \mu \mathrm{l}$ of water was rapidly drawn up and down 30 times (taking about 60 seconds) to rinse and agitate, and then repeated in the next two water rows. Then LC stain was drawn in and held for 10 minutes, followed by expelling and another $2 \times 15$ water rinses. The capsules were then rested on filter paper to drain off water, and removed and stored in the capsule storage box (which they came in) until TEM imaging.

Figures $2 \mathrm{a}$ and $2 \mathrm{~b}$ show images from two grids prepared by our standard droplet method. For diagnostic analyses these images are difficult to see fine detail due to some precipitates. Figures $3 \mathrm{a}$ and $3 \mathrm{~b}$ (from the same tissue blocks) were prepared using mPrep/g capsule staining. Stain intensity is similar but there is a noticeable reduction of precipitate. Thus, the mPrep/g staining provided more reliable images. While it is possible to re-rinse the poor droplet prepared grids to 
reduce the precipitate, this would substantially decrease efficiency and may damage the grid. In addition to providing more consistent results, $\mathrm{mPrep} / \mathrm{g}$ processing was also easier and more efficient. With mPrep/g processing, 16 grids were only handled when inserted into the capsules (and when put in the TEM). They were never damaged during staining, and since the capsules were labeled they were much easier to document. Further, since 16 grids were prepared simultaneously, the overall time and effort required was reduced by an order of magnitude since the time to prepare, store, document and stain each grid took only seconds compared to minutes using the droplet method [2].
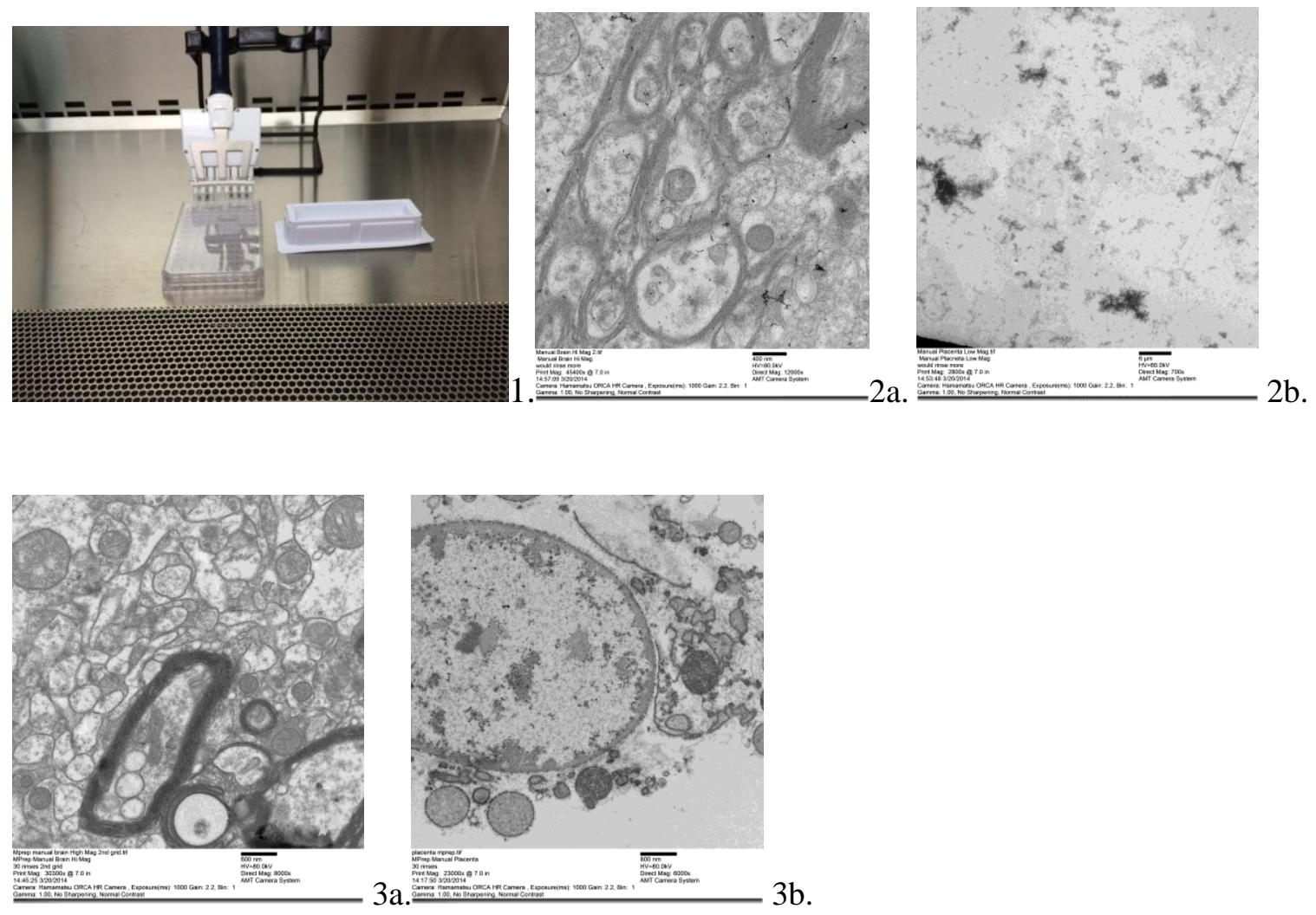

Figure 1. mPrep/g capsules on 8-channel Pipetman, and a 96 well plate used to hold reagents. Figure 2. a) Brain and b) Placenta tissues stained using the droplet method.

Figure 3. a) Brain and b) Placenta tissues stained using the mPrep/g system method.

[1] J Bozzola, L Russell, "Electron Microscopy: Principles and Techniques for Biologists", Jones and Bartlett Pub. MA, 1998 p. 120.

[2] Thanks to Steven Goodman and Tom Strader of Microscopy Innovations for suggestions and assistance with protocol development. 